\title{
Atmospheric Neutrino Fluxes*
}

\author{
Thomas K. Gaisser \\ Bartol Research Institute, University of Delaware \\ Newark, DE 19716 USA
}

November 18, 2018

\begin{abstract}
Starting with an historical review, I summarize the status of calculations of the flux of atmospheric neutrinos and how they compare to the measurements.
\end{abstract}

\section{Introduction}

When cosmic-ray protons and nuclei enter the atmosphere, they interact and produce all kinds of secondary particles, which in turn interact or decay or propagate to the ground, depending on their intrinsic properties and energies. In the GeV energy range, the most abundant particles at the ground are the neutrinos. At production, there are approximately twice as many muon neutrinos as electron neutrinos. This characteristic ratio arises from the pion-muon-electron decay chain in which a muon and one muon-neutrino (or anti-neutrino) are produced when the charged pion decays, while the subsequent muon decay produces an electron neutrino as well as another muon neutrino.

In 1960 Markov [1] suggested using Cherenkov light in a lake or the deep ocean to do neutrino physics with the atmospheric neutrino beam; in particular, to investigate the question whether electron and muon neutrinos are distinct species. About the same time Greisen [2] described a proposal to search for astrophysical sources of neutrinos with a water Cherenkov detector in a deep mine. Atmospheric neutrinos were, however, first detected with electronic detectors-as horizontal neutrinoinduced muons in a mine in South Africa [3] and in an iron calorimeter in a deep mine in the Kolar Gold Fields of India [4]. More than a decade passed before a largescale effort to instrument a large body of water was undertaken by the DUMAND Project [5].

Atmospheric neutrino fluxes were first calculated in the 1960s. One approach [6 is to infer the neutrino fluxes from measurements of closely related muons. More

${ }^{*}$ Research supported in part by the U.S. Department of Energy under DE-FG02 91ER40626. 
recently this approach has been refined to take account of effects of the geomagnetic field and of the separate contributions of pions and kaons [7. Most later calculations follow Refs. [8, 9] and calculate both the muon [8] and the neutrino fluxes [9] starting from the primary cosmic-ray spectrum at the top of the atmosphere.

With the advent in the 1980s of large underground detectors to search for proton decay, it became possible to measure increasingly large samples of events induced by neutrinos of both types (and from all directions because the Earth is transparent to neutrinos). Such events were of interest primarily as the background for proton decay. Both water Cherenkov detectors [10, 11] and segmented iron calorimeters [4, 12, 13, 14, were used.

Evidence gradually accumulated that the ratio of the two neutrino flavors was different from the expected value of two. The first hint came from the IMB experiment [15], which reported too few muon decays compared to what was expected from from interactions of $\nu_{\mu}$ inside their detector. The IMB experiment later concluded, however, that their measurement of the ratio of stopping to throughgoing muons was inconsistent with oscillations [16]. The Kamiokande experiment [17] found an anomalously low ratio of $\nu_{\mu} / \nu_{e}$ and suggested neutrino oscillations as a possible explanation. As the IMB experiment accumulated more data [18, they too found a persistently low $\nu_{\mu} / \nu_{e}$ flavor ratio. Meanwhile, however, the Frejus experiment [13] measured a ratio consistent with the expectation. After some six years of running with the shielded Kamiokande II-III detector, enough data was accumulated to see a suggestion of the pathlength dependence expected from oscillations with parameters such that upward $\nu_{\mu}$ in the multi-GeV energy region oscillate while downward neutrinos do not [19].

By measuring the directions and energies of thousands of neutrinos of both flavors in the past decade, the Super-Kamiokande Collaboration has shown that the anomalous ratio is a consequence of neutrino flavor oscillations during their propagation from the atmosphere to the detector [20]. They have measured the oscillations in the muon neutrino-tau neutrino sector [21] by fitting the energy and angular dependence and other observed features of their data, while ruling out oscillation to sterile neutrinos at $99 \%$ confidence level. It is now possible to demonstrate the $L / E$ pathlength/energy dependence expected for oscillations [22]. Best fit parameters in a two-flavor approximation $\left(\nu_{\mu} \leftrightarrow \nu_{\tau}\right)$ are $\delta m^{2}=2.1 \times 10^{-3} \mathrm{eV}^{2}$ and maximal mixing [23. Results from MACRO [24] and Soudan [14] are consistent with these parameters. Measurements of atmospheric neutrinos are reviewed in Refs. [25, 26, 27].

With the parallel discovery [28, 29, 30] of oscillations of solar electron-neutrinos in a different region of parameter space, a pattern of mixing among the three neutrino types (electron, muon and tau) is beginning to emerge in which two mixing angles are large while one is smaller (though not yet measured). Eventually the nature of the full neutrino mass matrix will be addressed with long-baseline accelerator neutrino beams by experiments now under construction or planned. This will take some time, however. Accordingly, there is interest in refining calculations of the 
atmospheric neutrino beam to the point where effects of sub-dominant mixing may be resolved. To take full advantage of the power of the Super-Kamiokande experiment requires reducing uncertainties in the absolute normalization of the neutrino flux and in the ratios of electron to muon neutrinos and neutrinos to anti-neutrinos. The normalization depends on the primary cosmic-ray intensity and on the details of production of pions and kaons in cosmic-ray interactions in the atmosphere. The ratios depend primarily on pion and kaon production. This paper reviews the present level of uncertainties.

\section{Calculation}

The neutrino flux is a convolution of the primary cosmic-ray spectrum, modified by geomagnetic cutoffs, with the yield of neutrinos per incident cosmic-ray. Schematically,

$$
\phi_{\nu}=\phi_{p} \otimes R_{p} \otimes Y_{p \rightarrow \nu}+\sum_{A} \phi_{A} \otimes R_{A} \otimes Y_{A \rightarrow \nu}
$$

The first term represents the contribution of free protons and the second term the contribution of nucleons bound in nuclei. The two need to be calculated separately even in the superposition approximation in which it is assumed that bound nucleons interact independently as if they were free. This is because the geomagnetic cutoff depends on rigidity (total momentum per unit charge) whereas pion production depends on energy per nucleon. In each term the factor $\phi(E)$ represents the primary cosmic-ray spectrum as a function of energy per nucleon. $R\left(E, \theta_{\oplus}, \phi_{\oplus}, \theta, \phi\right)$ indicates the cutoff rigidity (more exactly the transmission probability) which depends on latitude and longitude and on the local zenith and azimuth of the arrival direction of each primary cosmic ray. The yield $Y\left(E_{A}, E_{\nu}\right)$ gives the number of neutrinos with energy $E_{\nu}$ produced per primary of energy $E_{A}$ and is obtained by calculating the cosmic-ray induced cascades in the atmosphere. A complete review of the calculation of the flux of atmospheric neutrinos is given in Ref. 31. In this paper I illustrate the considerations involved in calculating the production spectrum of atmospheric neutrinos by comparing results of three recent calculations [32, 33, 34].

All these calculations are three-dimensional, taking account of the deviations of the neutrinos from the directions of the primaries that produce them. In addition, the calculations of Refs. 33 and 34 also account for the effect bending of muons in the geomagnetic field before they decay. There are several other fully three-dimensional calculations, including the recent papers of Refs. 35, 36, 37] which appeared after the review 31. The challenge of the full three-dimensional calculation is indicated by the five arguments of the cutoff function above. Cascades must be generated for relevant primary energies all over the globe $\left(\theta_{\oplus}, \phi_{\oplus}\right)$ taking account of the cutoff for primaries from all directions $(\theta, \phi)$. Since most of the created neutrinos miss the detector, the calculation is highly inefficient. Although

three-dimensional aspects of a calculation are technically challenging, they are not 
the most important sources of uncertainty in the calculated fluxes of atmospheric neutrinos. The biggest uncertainties come from the primary cosmic-ray spectrum and the treatment of hadronic interactions.

\subsection{Primary spectrum}

Primary spectra of nucleons

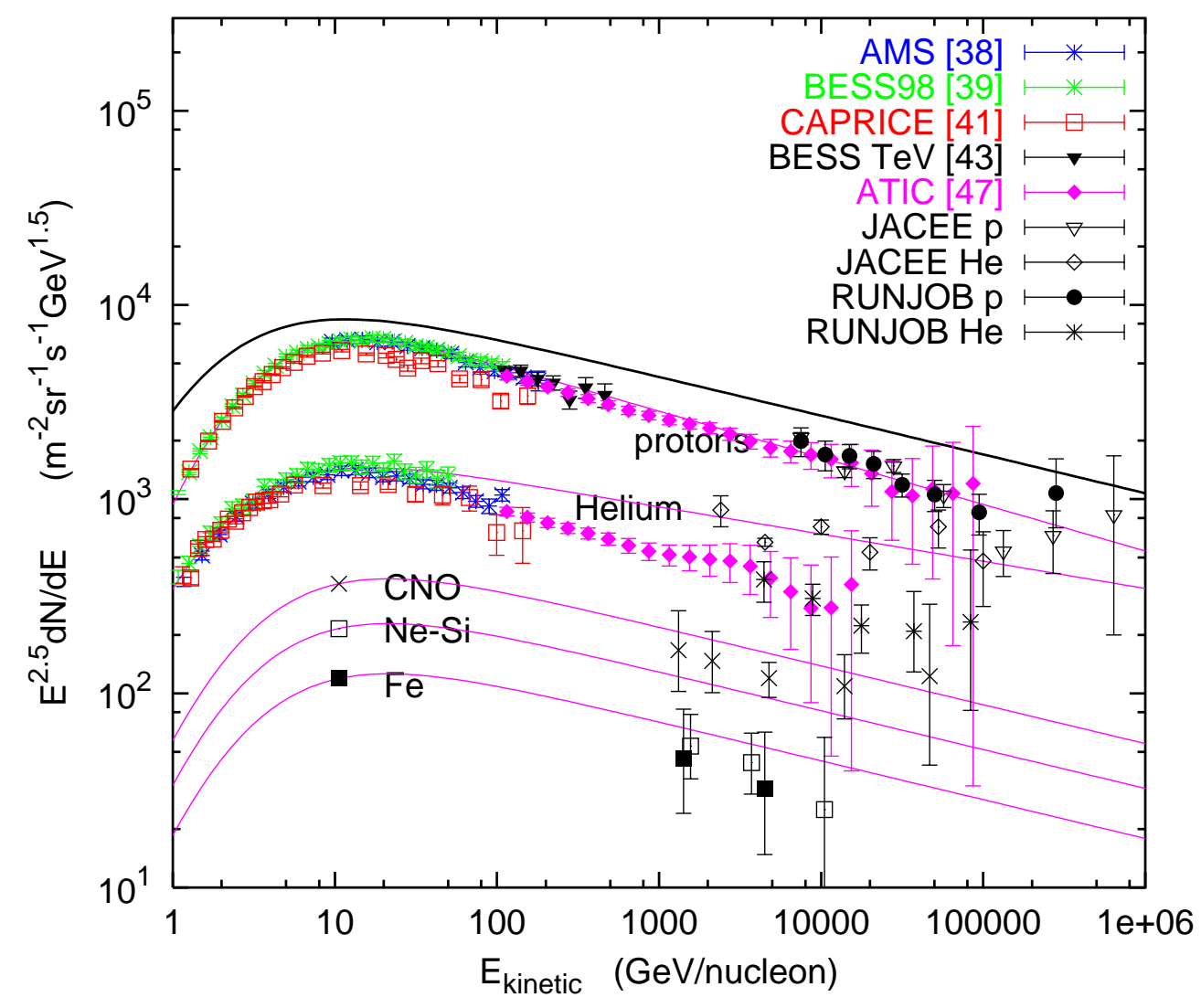

Figure 1: The flux of nucleons. The heavy black line shows the numerical form of Eq. 2. The lighter lines show extrapolations of fits [40] to measurements of protons, helium and three heavier groups below $100 \mathrm{GeV} /$ nucleon.

In the past decade new measurements of the primary spectrum with magnetic spectrometers have improved our knowledge of the primary spectrum. In particular, the AMS [38] and BESS [39] detectors give results for the proton spectra up to $100 \mathrm{GeV}$ that agree with each other within $5 \%$. As a consequence, current calculations of atmospheric neutrinos are using fits to the primary spectrum in which these data have been given priority. An example of such a fit [40] is shown by the thin lines in Fig. 1. Here the various nuclear groups are plotted as nucleons per $\mathrm{GeV} /$ nucleon, which is the quantity most directly related to secondary particle 
production. Free protons make up about $70 \%$ of the flux of all nucleons, helium about 20\%, and heavier nuclei the rest. Another recent series of spectrometer measurements (CAPRICE [41) continues to give results for protons about $15 \%$ lower than BESS and AMS, which can be taken as an indication of the uncertainty in the absolute normalization of this component of the primary spectrum. It should also be noted that BESS [39] and AMS [42] results for helium do not agree with each other as well as for protons.

Measurements with spectrometers presently extend up to about $500 \mathrm{GeV}$ only [43]. Higher energy data are from balloon-borne ionization calorimeters, which sample the fraction of energy deposited in electromagnetic cascades inside the calorimeter. Systematic errors in assigning primary energy are larger as a consequence. Data from JACEE [44] and RUNJOB [45, 46] are included in Fig. 11. Preliminary data from the ATIC experiment [47. fill in the gap in the $1-100 \mathrm{TeV}$ region. The ATIC data for protons appear consistent with all previous data. Their helium data, if confirmed, indicate a preference for the lower RUNJOB normalization for helium.

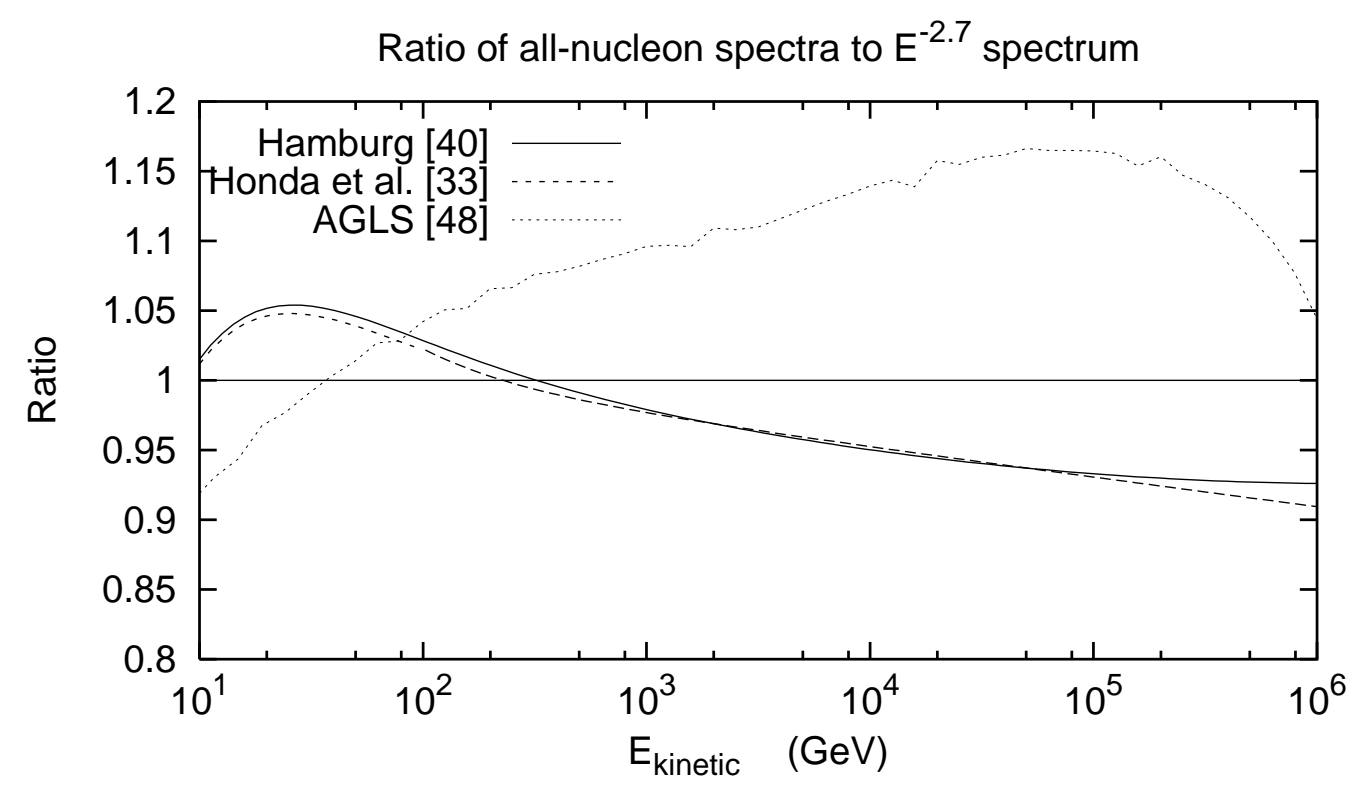

Figure 2: Ratio of the all-nucleon fluxes used in several calculations to the power-law form of Eq. 2.

The heavy solid line in Fig. 1 is a simple power-law approximation to the spectrum of all nucleons,

$$
\phi_{N}(E)=1.7 \frac{\text { nucleons }}{\mathrm{cm}^{2} s \mathrm{sr} G e V} \times\left(\frac{1}{E}\right)^{2.7},
$$

where $E$ is total energy per nucleon. Fig. 2 displays the all-nucleon spectra used to calculate the neutrino flux as a ratio to the power-law form of Eq. 2, Refs. 32, 34] use the fits of Ref. [40, while Ref. 33] use slightly different parameters for the 
heavy components at low energy and a slightly flatter power-law extrapolation (2.71) above $100 \mathrm{GeV}$. Nevertheless, the primary all-nucleon spectrum of the three calculations [32, 33, 34] are the same to within about 1\%. Moreover, simple power law approximation 2 to the all-nucleon spectrum represents these fits to better than $10 \%$ over the whole energy region. For reference, Fig. 2 also shows the primary spectrum used in an earlier calculation [48], which is somewhat lower than the new fits below $100 \mathrm{GeV}$ and significantly higher at high energy.
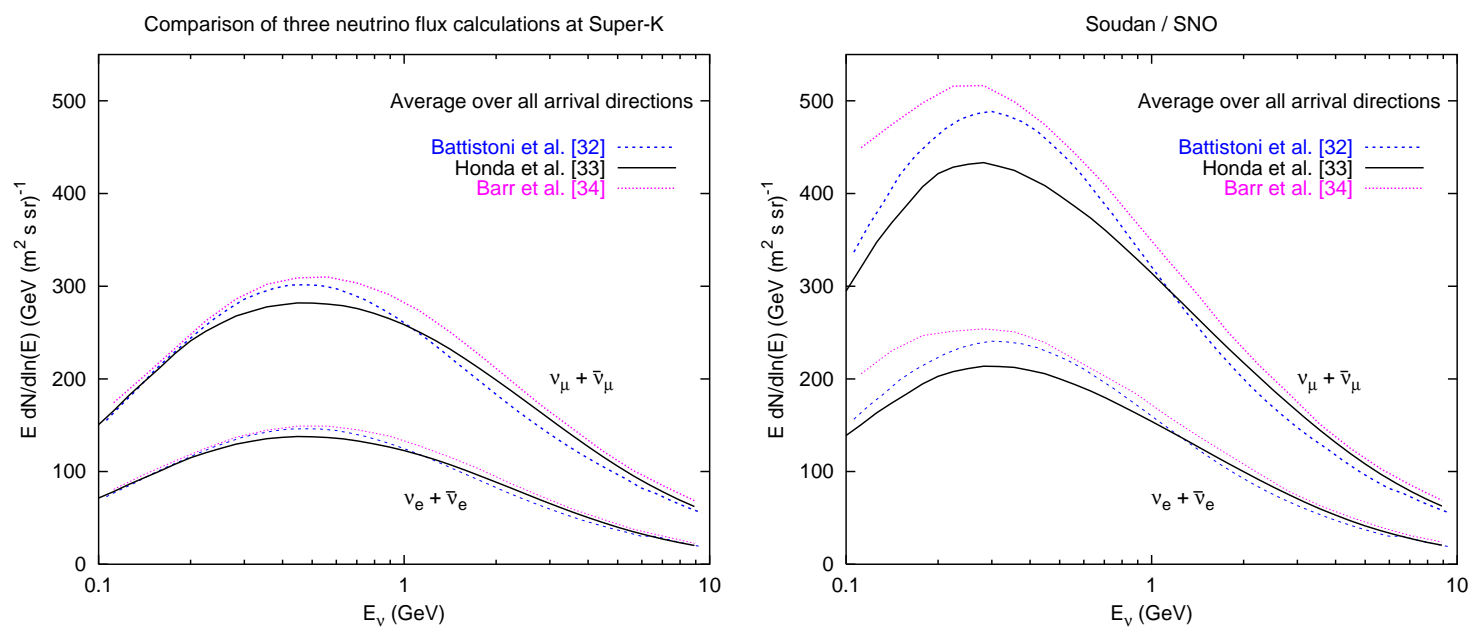

Figure 3: The production spectrum of $\nu_{\mu}+\bar{\nu}_{\mu}$ and $\nu_{e}+\bar{\nu}_{e}$ integrated over the atmosphere and averaged over all directions at Super-K (left) and Soudan or SNO (right).

\subsection{Treatment of hadronic interactions}

The representations of hadronic interactions used in Refs. 32, 33, 34, are completely independent. As a consequence, it is not surprising that they give different results. Because the assumptions about primary spectrum are essentially identical, differences among the results of the three calculations reflect the level of uncertainty due to treatment of hadronic interactions. For low energies the fluxes depend also on the geomagnetic cutoffs, so comparison must be made for the same location. Figure 3 shows the atmospheric neutrino fluxes from the three calculations [32, 33, 34]. Differences among the calculations are at the level of $10 \%$ at Super-K. The differences are somewhat larger for the sites at high geomagnetic latitude (where the cutoff for downward primaries is negligible), which probably indicates that the low-energy hadronic interactions are less well understood.

The flavor ratios for the three calculations are shown for the low-energy region in Fig. 4, along with the neutrino/antineutrino ratios. Differences are at the level of $3 \%$ in this energy range for the flavor ratios and $5 \%$ for the $\nu / \bar{\nu}$ ratios. 

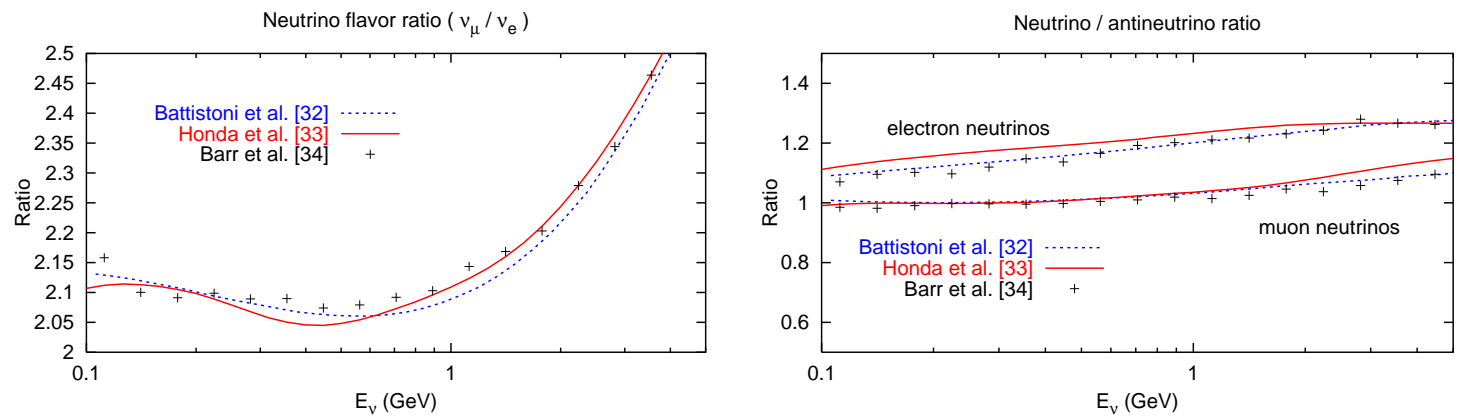

Figure 4: The ratio $\nu_{\mu}+\bar{\nu}_{\mu}$ to $\nu_{e}+\bar{\nu}_{e}$ (left panel) and $\nu / \bar{\nu}$ (right panel) at Super-K. The production spectra are integrated over the atmosphere neglecting oscillations and averaged over all directions before taking the ratio.
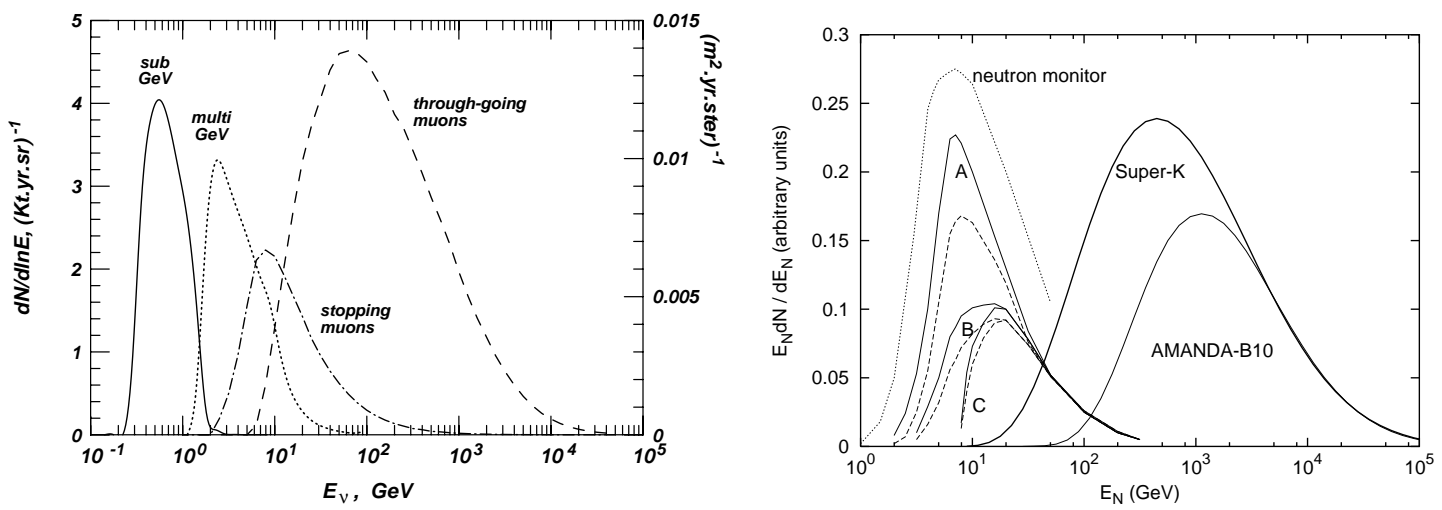

Figure 5: Distribution of neutrino energies (left) and primary energy per nucleon (right) giving rise to various classes of events.

\section{$3 \quad$ Higher energies}

Response functions for various measurements of atmospheric neutrinos are given in Ref. 31] and reproduced here in Fig. 5. Sub-GeV and multi-GeV events involve neutrinos with energies below $2 \mathrm{GeV}$ and from 1 to $20 \mathrm{GeV}$ respectively. Upward stopping muons are produced by muon neutrinos with energies in the range 3-100 $\mathrm{GeV}$, while upward throughgoing muons come from neutrinos with energies in the range $10 \mathrm{GeV}$ to $10 \mathrm{TeV}$. The relevant primary energies per nucleon are roughly a factor of ten higher, but with rather broader distributions. Thus the normalization of the sub-GeV neutrinos is determined almost entirely by primaries with energies less than $100 \mathrm{GeV} /$ nucleon and hence covered by the most precise magnetic spectrometer measurements. On the other hand, the primary response function for neutrinoinduced muons extends to $10 \mathrm{TeV}$ and beyond and hence is subject to somewhat larger uncertainty in normalization. The difference in response function for neutrinoinduced upward muons at Super-K from that at AMANDA arises from the higher 
muon energy threshold in AMANDA, which is of order $100 \mathrm{GeV}$ at the interaction vertex 49.

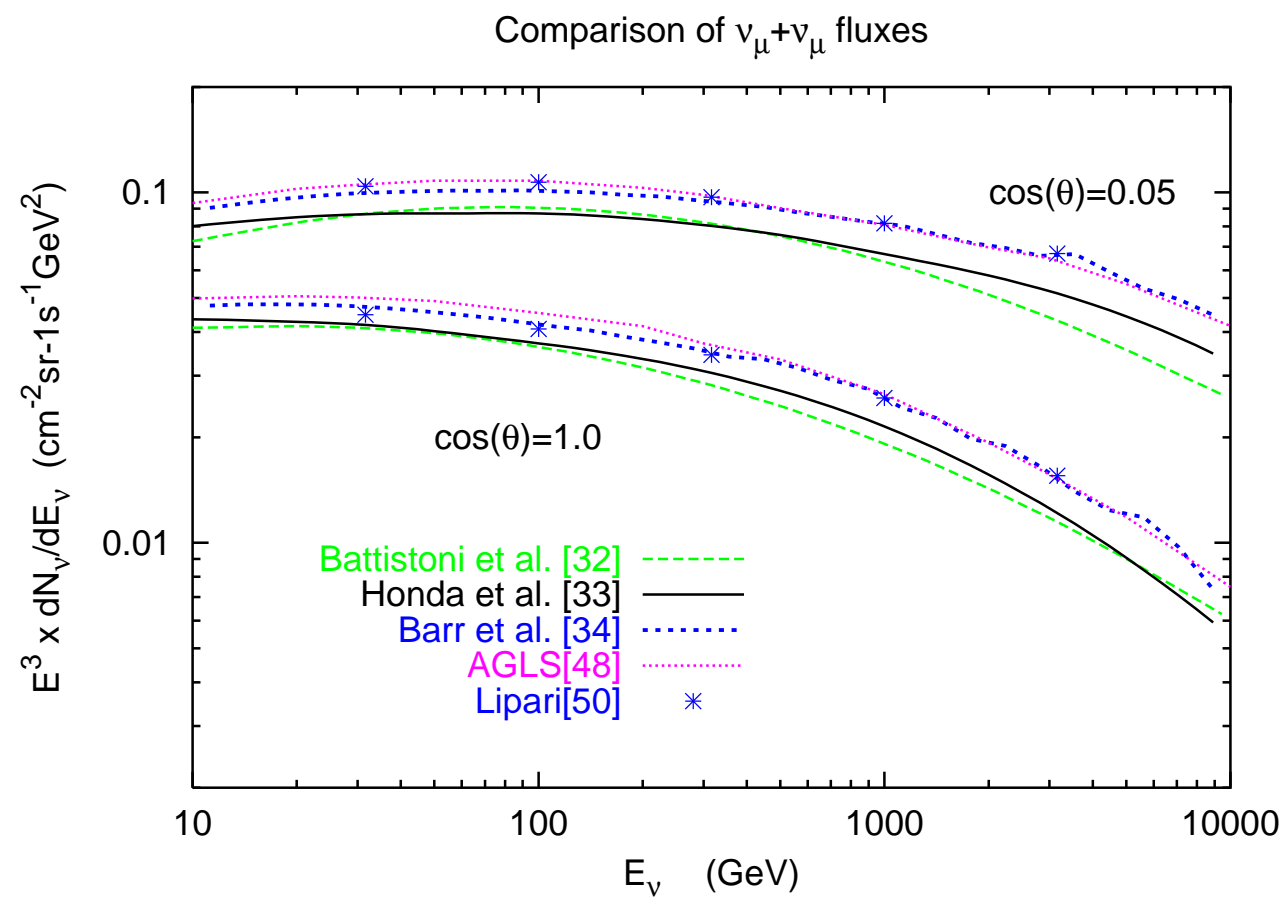

Figure 6: Comparison of fluxes of muon neutrinos at high energy.

Again we can make use of the fact that the assumed primary spectra in the three calculations [32, 33, 34] are almost the same to illustrate differences due to the treatment of hadronic interactions. Fig. 6] compares fluxes of muon neutrinos for these three calculations in the energy range responsible for neutrino-induced muons.

\subsection{Analytic approximations}

At high energy, numerical integration of the analytic cascade equations gives sufficiently accurate results to be useful for understanding the characteristic features of the results. The asterisks in Fig. 6] show the numerical results of Lipari [50, including the contribution from decay of muons. ${ }^{1}$ At high energy most muons reach the ground before decaying and therefore do not contribute to the neutrino flux. For $E_{\nu}>100 \mathrm{GeV}$ for example, less than $15 \%$ of muon neutrinos are from decay of muons, and the fraction decreases further as energy increases.

Neglecting neutrinos from muon decay, a simple approximation for the flux of $\nu_{\mu}+\bar{\nu}_{\mu}$ from decay of pions and kaons is

$$
\frac{d N_{\nu}}{d E_{\nu}}=\frac{\phi_{N}\left(E_{\nu}\right)}{\left(1-Z_{N N}\right)(\gamma+1)}\left\{\left[\frac{Z_{N \pi}\left(1-r_{\pi}\right)^{\gamma}}{1+B_{\pi \nu} \cos \theta E_{\nu} / \epsilon_{\pi}}\right]\right.
$$

\footnotetext{
${ }^{1}$ The primary spectrum of [50] has the same form as Eq. 2 with a higher normalization.
} 


$$
\left.+0.635\left[\frac{Z_{N K}\left(1-r_{K}\right)^{\gamma}}{1+B_{K \nu} \cos \theta E_{\nu} / \epsilon_{K}}\right]\right\},
$$

where $\phi_{N}\left(E_{\nu}\right)$ is the primary spectrum of nucleons evaluated at the energy of the neutrino (see Eq. 2). The constants $r_{i}=m_{\mu}^{2} / m_{i}^{2}$ for $i=(\pi, K)$, while the constants $B$ depend on the hadron attenuation lengths as well as decay kinematics. The critical energy for pions is $\epsilon_{\pi} \approx 115 \mathrm{GeV}$, while for kaons $\epsilon_{K} \approx 850 \mathrm{GeV}$ [51].

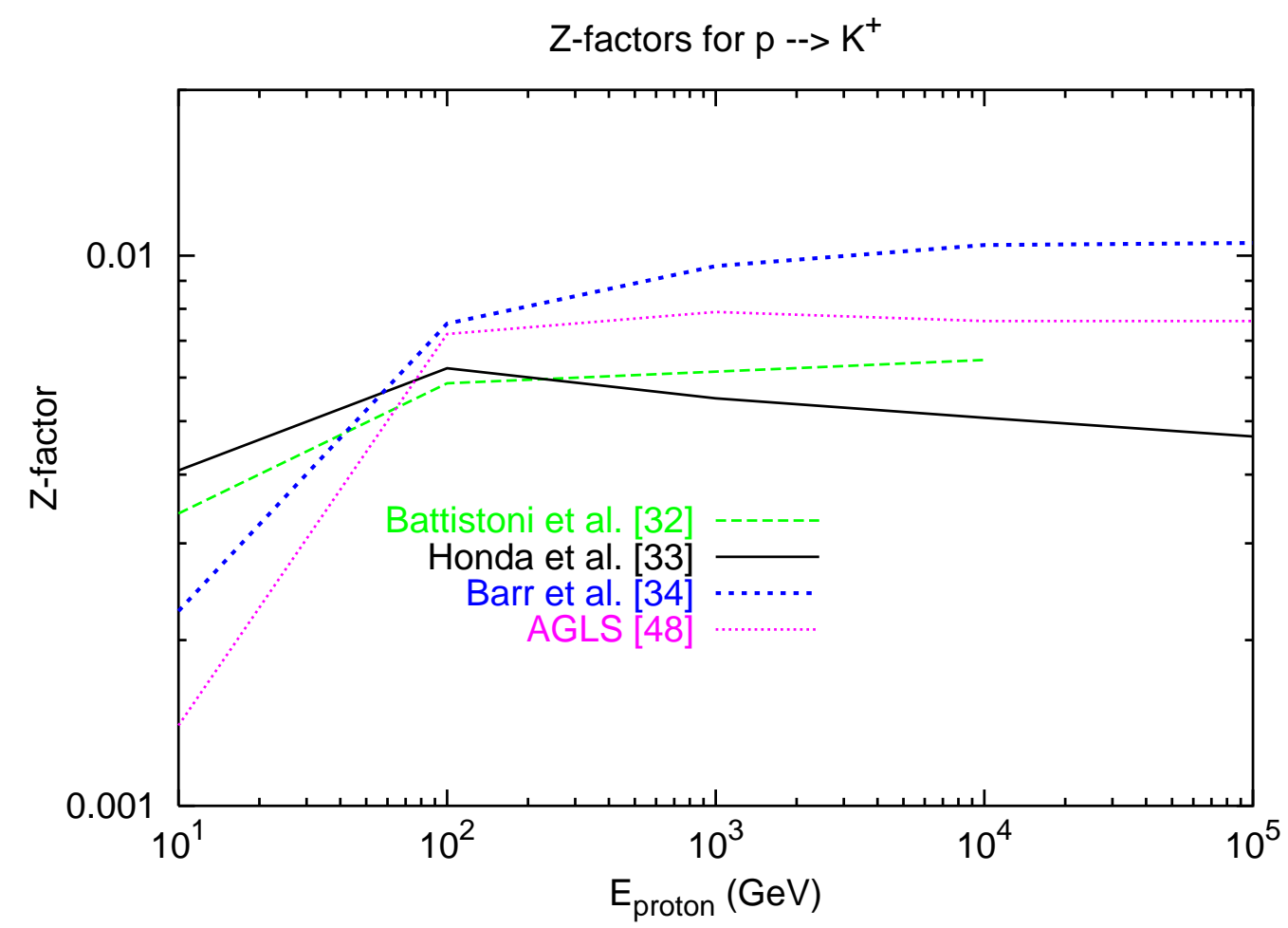

Figure 7: Spectrum-weighted Z-factor for $K^{+}$production by nucleons.

Asymptotically, the neutrino spectrum is one power steeper than the primary spectrum. For fixed zenith angle $\theta$ the transition occurs first for neutrinos from pion decay because of its lower critical energy. As a consequence, the relative contribution from kaons increases with energy. Kaons become the dominant source of neutrinos for $E_{\nu}>100 \mathrm{GeV}$. (The onset of kaon dominance is somewhat slower at large angle because of the cosine factor in the denominator of the kaon term in Eq. 3.) Important differences among the calculations can be traced to differences in treatment of kaon production, in particular to the channel $p \rightarrow \Lambda K^{+}$. Fig. [7 shows the spectrumweighted moments [51] for four calculations.

Several features of Fig. 6] can be understood with the help of Eq. 3]

- The general decrease of the vertical to horizontal ratio at large energy, which appears in all calculations, is a consequence of the factor of $\cos (\theta)$ in the denominators. 
- The primary spectrum of [34] is significantly lower than that of [48] at high energy, as shown in Fig. 2. The calculation of Ref. [34] is nevertheless high, comparable to that of Ref. [48] because the kaon production assumed in the new calculation is larger, as shown in Fig. 7.

- The ratio $(>1)$ of the flux of Ref. 34 compared to that of Ref. [33, and its increase with energy, may also be attributed to the differences in the assumptions for kaon production as shown in Fig. 7.

\subsection{Muon fluxes}

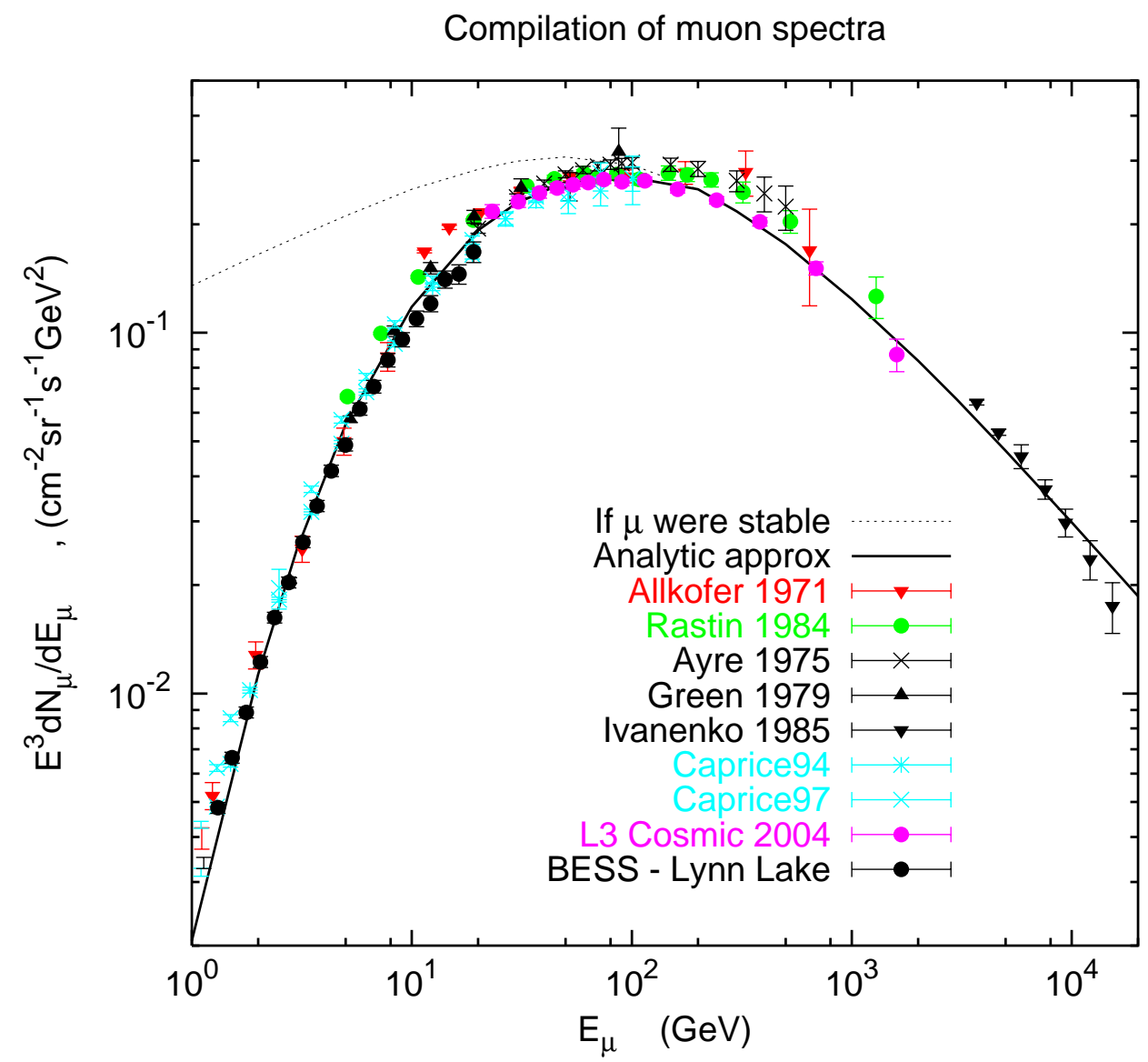

Figure 8: Summary of measurements of the vertical muon intensity at the ground. The solid line shows an analytic calculation [52. The dotted line shows the spectrum in the absence of decay and energy loss, or equivalently the muon production spectrum integrated over the atmosphere.

Calculation of the flux of atmospheric muons follows the same form as Eq. 1 with the yield of neutrinos replaced by the yield of muons. Because of the close 
relation between muons and neutrinos, comparison of its predicted muon intensity to measurements is an important check on any calculation of atmospheric muons. Comparison to muon fluxes also serves to check the normalization of the calculation. Because muons lose about $2 \mathrm{GeV}$ in passing through the atmosphere, a ground level measurement probes high energies and is therefore most relevant to multi-GeV neutrinos and to neutrino-induced upward muons. Because of the kinematics of the $\pi \rightarrow \mu$ decay, however, pion decay remains the dominant source of muons at all energies. As a consequence, the leverage of the muon measurements for controlling the neutrino flux is limited above $\sim 100 \mathrm{GeV}$ where neutrino production is dominated by the contribution from kaons.

Fig. 8 contains a summary of measurements of the spectrum of vertical muons at the ground. All measurements have been corrected to sea level. At very high energy the muon spectrum becomes one power steeper than the parent spectrum of nucleons as a consequence of the extra power of $1 / E_{\pi}$ in the ratio of pion decay length to interaction length, which reflects the decreasing probability of decay relative to reinteraction for charged pions at high energy. For $E<\epsilon_{\pi} \approx 115 \mathrm{GeV}$ essentially all pions decay, and the muon production spectrum has the same power behavior as the parent pion and grandparent nucleon spectrum $(\alpha \approx 2.7)$. At low energy, however, muon energy-loss and decay become important, and the muon spectrum at the ground falls increasingly below the production spectrum. To account for all the complications one generally resorts to Monte Carlo calculations. However, analytic approximations of the effects are also possible [50. Fig. 8] shows a comparison of one such calculation [52], which uses as input the simple power-law primary spectrum of Eq. 2. Data in Fig. 8 are from many measurements with spectrometers at the surface [53, 54, 55, 56, 57, 58, and underground [59, 60].

The comparison of the analytic calculation with the data in Fig. 8 leads to two remarks. First the good agreement gives a general confirmation of the primary spectrum at high energy. Second, it serves to illustrate the level of systematic differences among the various measurements.

\section{Concluding remarks}

In their recent detailed paper, 23] the Super-Kamiokande group give an extensive discussion of how the best fit oscillation parameters are determined from a comparison of their data with simulations based on the atmospheric neutrino flux of Ref. 33. In particular, their Table VII enumerates the assumed uncertainties in various properties of the calculated neutrino flux and the shifts in those properties needed to obtain the best fit. Apart from the overall increase in normalization, the largest shifts relative to what is assumed in Ref. 33] concern the slope of the primary spectrum at high energy and the $\mathrm{K} / \mathrm{pi}$ ratio. The latter is decreased by $6 \%$ overall, while the primary spectrum is significantly harder as compared to the fits of Ref. 40], (from -2.71 to -2.66 above $100 \mathrm{GeV}$ ). As noted in the discussion of 
Figs. 6 and 7 , the high energy $\nu$-spectrum could also be increased by enhancing kaon production preferentially at high energy.

The Super-K fitting procedure uses the atmospheric neutrino data itself to correct the calculated neutrino flux. The neutrino data have the advantage in principle that (apart from oscillations!) the intensity observed at the detector integrates over the whole atmosphere without complications of energy loss and decay that dominate the observed muon flux. For example the required increase in the overall normalization of $11.9 \%$ could be interpreted as favoring the higher normalization of Refs. [38, 39] as compared to that of Ref. [41. The observations depend, however, on properties of neutrino interactions as well as on the neutrino flux. As properties of neutrino interactions become better determined, for example from near detectors of long-baseline experiments, this iterative procedure may become an important way to refine our understanding of the atmospheric neutrino flux at production.

Acknowledgments. I am grateful to G. Battistoni and M. Honda for providing the Z-factors for their hadronic interaction models and to G. Barr, G. Battistoni, M. Honda, T. Kajita, P. Lipari, S. Robbins and T. Stanev for helpful discussions.

\section{References}

[1] Markov, M.A., Proc. 1960 Annual International Conference on High Energy Physics at Rochester (ed. E.C.G. Sudarshan, J.H. Tinlot \& A.C. Melissinos, Rochester) 578 (1960).

[2] Greisen, K. Ann. Revs. Nuclear Science 10, 63 (1960).

[3] Reines, F. et al., Phys. Rev. Letters 15, 429 (1965).

[4] Achar, C.V. et al., Phys. Lett. 18, 196 (1965).

[5] Babson, J. et al., Phys. Rev. D 42, 3613 (1990).

[6] Markov, M.A. \& Zheleznykh, I.M., Nucl. Phys. 27385 (1961).

[7] Perkins, D.H., Astropart. Phys. 2, 249 (1994) and references therein.

[8] Zatsepin, G.T. \& Kuz'min, V.A. Sov. Phys. JETP 12, 1171 (1961).

[9] Zatsepin, G.T. \& Kuz'min, V.A. Sov. Phys. JETP 14, 1294 (1962).

[10] Becker-Szendy, R. et al., Phys. Rev. D 42, 2974 (1990).

[11] Hirata, K.S. et al., Phys. Rev. D 38, 448 (1988).

[12] Aglietta, M. et al., Europhys. Lett. 8, 611 (1989).

[13] Berger, Ch. et al., Phys. Lett. B 227, 489 (1989). and B 245, (1990). See also K. Daum et al., Z. Phys. C 66, 417 (1995). 
[14] Sanchez, M. et al., Phys. Rev. D 68, 113004 (2003).

[15] Haines, T.J. et al., Phys. Rev. Letters 57, 1986 (1986).

[16] Becker-Szendy, R. et al., Phys. Rev. Letters 69, 1010 (1992).

[17] Hirata, K.S. et al., Phys. Lett. B 205, 416 (1988)

[18] Becker-Szendy, R. et al., Phys. Rev. D 46, 3720 (1992) and references therein.

[19] Fukuda, Y. et al., Phys. Lett. B 335, 237 (1994).

[20] Fukuda, Y. et al., Phys. Rev. Lett. 81, 1562 (1998).

[21] Fukuda, S. et al., Phys. Rev. Letters 85, 3999 (2000).

[22] Ashie, Y. et al., Phys. Rev. Letters 93, 101801 (2004).

[23] Ashie, Y. et al., hep-ex/0501064.

[24] Ambrosio, M. et al., Phys. Lett. B566, 35 (2003).

[25] Kajita, T. \& Totsuka, Y., Revs. Mod. Phys. 73, 85 (2001).

[26] Jung, C.K., Kajita, T., Mann, T. \& McGrew, C., Ann. Revs. Nucl. Part. Sci., 51, 451 (2001).

[27] Goodman, M. Phys. Lett. B (Proc. Suppl.) 118, 99 (2002).

[28] Ahmad, Q.R. et al., Phys. Rev. Letters 87, 071301 (2001).

[29] Smy, M. et al., Phys. Rev. D 69, 011104 (2004).

[30] Ahmad, Q.R. et al., Phys. Rev. Letters 89, 011301 and 011302 (2002).

[31] Gaisser, T.K. \& Honda, M., Ann. Revs. Nucl. Part. Sci. 52, 153 (2002).

[32] Battistoni, G. et al., Astropart. Phys. 19, 269 (2003) (Erratum pp 291-294). http://www.mi.infn.it/ battist/neutrino.html

[33] Honda, M. et al., Phys. Rev. D 70, 043008 (2004).

http://www.icrr.u-tokyo.ac.jp/ mhonda/

[34] Barr, G.D. et al., Phys. Rev. D 70, 023006 (2004).

http://www-pnp.physics.ox.ac.uk/ barr/fluxfiles/

[35] Wentz, J. et al., Phys. Rev. D 67, 073020 (2003).

[36] Liu, Y. et al., Phys. Rev. D 67, 073022 (2003).

[37] Favier, J. et al., Phys. Rev. D 68, 093006 (2003). 
[38] Alcarez, J. et al., Phys. Lett. B490, 27 (2000).

[39] Sanuki, T. et al., Ap.J. 545, 1135 (2000).

[40] Gaisser, T.K., Honda, M., Lipari, P. \& Stanev, T., Proc. 27th Int. Cosmic Ray Conf., (Hamburg) 5, 1643 (2001).

[41] Boezio, M. et al., Ap.J. 518, 457 (1999).

[42] Alcarez, J. et al., Phys. Lett. B 494, 193 (2000).

[43] Haino, S. et al., astro-ph/0403704.

[44] Asakimori, K. et al. Ap.J. 502, 278 (1998).

[45] Apanasenko, A.V. et al., Astropart. Phys. 16, 13 (2001).

[46] Furukawa, M. et al., Proc. 28th Int. Cosmic Ray Conf. (Tsukuba) 4, 1837 (2003).

[47] Wefel, J. et al., in Proc. 28th Int. Cosmic Ray Conf. (Tsukuba) 4, 1849 (2003). Preliminary ATIC data are from the presentation of H. Ahn at COSPAR 2004 (Paris).

[48] Agrawal, V., Gaisser, T.K., Lipari, P., \& Stanev, T., Phys. Rev. D53, 1314 (1996).

[49] Ahrens, J. et al., Phys. Rev. D 66, 012005 (2002).

[50] Lipari, P., Astropart. Phys. 1, 195 (1993).

[51] Gaisser, T.K. Cosmic Rays and Particle Physics (Cambridge University Press, 1990).

[52] Gaisser, T.K., Astropart. Phys. 16, 285 (2002).

[53] O.C. Allkofer, O.C., Carstensen, K. \& Dau, W.D., Phys. Lett. B36, 425 (1971).

[54] Rastin, B.C., J. Phys. G 10, 1609 (1984).

[55] Ayre, C.A. et al., J. Phys. G 1 (1975) 584.

[56] Green, P.J. et al., Phys. Rev. D 20, 1598 (1979).

[57] Kremer, J. et al. (CAPRICE) Phys. Rev. Letters 83, 4241 (1999).

[58] Motoki, M. et al. (BESS) Astropart. Phys. 19, 113 (2003).

[59] Achard, P. et al., (L3) Phys. Lett. B 598, 15 (2004).

[60] I.P. Ivanenko, I.P., et al., Proc. 19th Int. Cosmic Ray Conf., La Jolla (NASA Conf. Publ. No 2376) 8, 210 (1985). 